\title{
Controllability and controller-observer design for a class of linear time-varying systems
}

\begin{abstract}
In this paper a class of linear time-varying control systems is considered. The time variation consists of a scalar time-varying coefficient multiplying the state matrix of an otherwise time-invariant system. Under very weak assumptions of this coefficient, we show that the controllability can be assessed by an algebraic rank condition, Kalman canonical decomposition is possible, and we give a method for designing a linear state-feedback controller and Luenberger observer.
\end{abstract}

Keywords Linear time-varying systems · Controllablility · Kalman canonical structure $\cdot$ Stabilisation $\cdot$ Observer

\section{Introduction}

Consider the following linear time-varying control system:

$$
\left\{\begin{array}{l}
\dot{x}(t)=a(t) A x(t)+B u(t) \\
y(t)=C x(t) \\
x(0)=x_{0}
\end{array}\right.
$$

We assume that the following data are given:

$$
A \in \mathbb{R}^{n \times n}, \quad B \in \mathbb{R}^{n \times m}, \quad C \in \mathbb{R}^{p \times n} .
$$

We further assume that the function $a:[0, T] \rightarrow \mathbb{R}$ is a given integrable function. The state equation of system (1) has been considered in [11]. There, under one further constraint on $a(t)$, it was shown that the complete controllability of (1) at a certain time $T$ is equivalent to the complete controllability of the pair $[A, B]$. This is a rather surprising result, given that the coefficient $a(t)$

Address(es) of author(s) should be given 
can change in an almost arbitrary manner and no smoothness assumptions are placed on it.

In this paper, we further explore the fundamental properties of (1), where we have included the possibility that only the output can be observed. In section 2 , we improve on the conclusion of [11] by showing that the complete controllability of (1) is indeed equivalent to the complete controllability of the pair $[A, B]$, under further weaker conditions on $a(t)$. This result is used in section 3 to show that a Kalman canonical system decomposition is possible for all time $t$. In section 4 , and based on the canonical decomposition, we give necessary and sufficient conditions for stabilisability/detectability, as well as a method for designing a stabilising linear state-feedback controller and a full order $\mathrm{Lu}-$ enberger observer.

Before we conclude this introduction, let us point out that a slightly more general system

$$
\left\{\begin{array}{l}
\dot{x}(t)=a(t) A x(t)+B B_{1}(t) u(t), \\
y(t)=C_{1}(t) C x(t), \\
x(0)=x_{0}, \\
B_{1}(\cdot), B_{1}^{-1}(\cdot) \in L^{\infty}\left([0, \infty) ; \mathbb{R}^{m \times m}\right), \quad C_{1}(\cdot), C_{1}^{-1}(\cdot) \in L^{\infty}\left([0, \infty) ; \mathbb{R}^{p \times p}\right),
\end{array}\right.
$$

can always be transformed into (1) by introducing a new control variable $u_{1}(t) \equiv B_{1}(t) u(t)$, and a new output variable $y_{1}(t) \equiv C_{1}^{-1}(t) y(t)$. Thus, all results of this paper are also valid for this more general system.

\section{Controllability}

Controllability and criteria for assessing it were introduced by Kalman in [7]. In general these criteria require either the calculation of the Grammian matrix or the solution of the Lyapunov matrix differential equation, neither of which is easy. Attempts have been made to obtain simpler criteria for assessing this fundamental system property. When the system coefficients have a certain number of derivatives or are analytic, then algebraic criteria that do not require solving the Lyapunov equation are available [3], [21], [19], [20]. In [11] an algebraic criterion for controllability of (1) is introduced that does not require the differentiability of $a(t)$. However, it imposes the constraint on the integral of $a(t)$ not being zero in the interval of interest. In this section, we prove that such an algebraic criterion is valid without the mentioned restriction. Indeed, apart from the minimum requirement of $a(t)$ being an integrable function, we make no further assumptions on it. Let us first recall the definition of complete controllability.

Definition 1 System (1) is called completely controllable at time $T>0$, if for each pair $x_{0}, x_{T} \in \mathbb{R}^{n}$, there exists a control $u(t)$ such that $x(T)=x_{T}$. 
In [11], the following rank condition for controllability of (1) at time $T$ is proved.

Theorem 1 Let the coefficient $a(\cdot)$ be such that

$$
\int_{0}^{T} a(t) d t \neq 0 .
$$

Then system (1) is completely controllable at time $T$ if and only if:

$$
\operatorname{rank} \mathcal{C}=n .
$$

where $\mathcal{C} \equiv\left[B, A B, \ldots, A^{n-1} B\right]$.

As pointed out in [11], the above theorem does not require for the coefficient $a(t)$ to be $n-2$ times differentiable, which is an advantage over the controllability criterion of [3], [21], [19], [20]. However, the opposite is also true: the systems with $n-2$ times differentiable coefficient $a(t)$, that do not satisfy condition (2), can be considered by the criterion of [3], [21], [19], [20]. Clearly, it is desirable to remove the constraint (2).

Let us recall the general necessary and sufficient condition for complete controllability as given in [7].

Lemma 1 System (1) is completely controllable at time $T$ if and only if:

$$
\operatorname{rank} G_{T}=n \text {. }
$$

Here $G_{T}$ is the controllability Gramian defined as:

$$
G_{T}=\int_{0}^{T} \Phi(t) B B^{\prime} \Phi^{\prime}(t) d t,
$$

where $\Phi(\cdot)$ is the solution to the following matrix differential equation:

$$
\left\{\begin{array}{l}
\dot{\Phi}(t)=a(t) A \Phi(t), \\
\Phi(0)=I
\end{array}\right.
$$

In [11] it is shown that $\Phi(t)=e^{g(t) A}$, where $g(t)=\int_{0}^{t} a(s) d s$. Then it is obvious that

$$
\Phi(t) a(t) A=a(t) A \Phi(t)
$$

Lemma 2 Let $a(t) \neq 0$, for a.e. $t \in[0, T]$. Then the system (1) is not completely controllable at time $T$, if and only if there exists a non-zero vector $p \in \mathbb{R}^{n}$ such that:

$$
p^{\prime} \Phi(t) A^{i} B=0, \quad \forall t \in[0, T], \quad i \in \mathbb{N}_{0} .
$$


Proof (Sufficiency.) Let the equations (7) hold, which in particular means that $p^{\prime} \Phi(t) B=0$. This implies $p^{\prime} G_{T} p=0$, i.e. the system is not completely controllable at time $T$.

(Necessity.). We prove this by induction. Assume that system (1) is not completely controllable at time $T$. Then there exists a non-zero vector $p \in \mathbb{R}^{n}$ such that $p^{\prime} G_{T} p=0$. Due to the nonnegativity of $G_{T}$, this implies:

$$
p^{\prime} \Phi(t) B=0, \quad \forall t \in[0, T] .
$$

This proves (7) for $i=0$. We assume that (7) holds for $i=0, \ldots, j \geq 0$, i.e.

$$
p^{\prime} \Phi(t) A^{j} B=0, \quad \forall t \in[0, T] .
$$

Now we need to prove that (7) holds for $i=j+1$. The derivative of $p^{\prime} \Phi(t) A^{j} B$ is:

$$
\frac{d}{d t}\left[p^{\prime} \Phi(t) A^{j} B\right]=p^{\prime} a(t) A \Phi(t) A^{j} B .
$$

Integration and commutation (6) give:

$$
p^{\prime} \Phi(t) A^{j} B=p^{\prime} A^{j} B+\int_{0}^{t} p^{\prime} \Phi(\tau) a(\tau) A^{j+1} B d \tau,
$$

which when substituted into (8) results in

$$
p^{\prime} A^{j} B+\int_{0}^{t} p^{\prime} \Phi(\tau) a(\tau) A^{j+1} B d \tau=0, \quad \forall t \in[0, T] .
$$

Taking $t=0$ in (9) implies that $p^{\prime} A^{j} B=0$, and thus equation (9) becomes

$$
\int_{0}^{t} p^{\prime} \Phi(\tau) a(\tau) A^{j+1} B d \tau=0, \quad \forall t \in[0, T] .
$$

Let $F(t)$ be defined as

$$
F(t) \equiv \int_{0}^{t} p^{\prime} \Phi(\tau) a(\tau) A^{j+1} B d \tau, \quad \forall t \in[0, T]
$$

Then (10) implies that $F(t)=0, \forall t \in[0, T]$, and hence is differentiable with derivative

$$
\frac{d F(t)}{d t}=0, \quad \forall t \in[0, T] .
$$

From Theorem 4.4.2 of [1], we have $p^{\prime} \Phi(t) a(t) A^{j+1} B=\frac{d F(t)}{d t}$, a.e. $t \in[0, T]$. Therefore,

$$
p^{\prime} \Phi(t) a(t) A^{j+1} B=0, \quad \text { a.e. } \quad t \in[0, T] .
$$

Due to our assumption on $a(\cdot)$, equation (12) is equivalent to:

$$
p^{\prime} \Phi(t) A^{j+1} B=0, \quad \forall t \in[0, T] .
$$

This confirms (7) for $i=j+1$, and thus completes the proof. 
We can now state and prove the main result of this section, where we have excluded the trivial case of $a(t)=0$ for a.e. $t \in[0, T]$.

Theorem 2 System (1) is completely controllable at time $T$ if and only if:

$$
\operatorname{rank} \mathcal{C}=n .
$$

Proof The proof proceeds in two parts. In part (a) we prove the theorem under the assumption that $a(t) \neq 0$, for a.e. $t \in[0, T]$. In part (b) we use this result to prove the general case.

(a) Let $a(t) \neq 0$, for a.e. $t \in[0, T]$.

(Sufficiency.) Assume that condition (13) holds, but the system is not completely controllable at time $T$. From Lemma 2 we know that there exists a non-zero vector $p \in \mathbb{R}^{n}$ such that

$$
p^{\prime} \Phi(t) A^{j} B=0, \quad \forall t \in[0, T], \quad j \in \mathbb{N}_{0} .
$$

The first $n$ equations in (14) are equivalent to:

$$
p^{\prime} \Phi(t)\left[B, A B, \ldots, A^{n-1} B\right]=0 .
$$

However, due to our assumption that (13) holds, this is not possible. Hence, the system is completely controllable.

(Necessity.) Assume that the system is completely controllable at time $T$, but (13) does not hold. This means that there exists a non-zero vector $p \in \mathbb{R}^{n}$, such that (15) holds, which in turn implies (14). From Lemma 2 we conclude that the system is not completely controllable at time $T$, and hence the contradiction.

(b) Let there be at least one interval $[a, b] \subset[0, T]$ such that $a(t) \neq 0$ for a.e. $t \in[a, b]$. Let there be $N$ disjoint subintervals of $[0, T]$, excluding $[a, b]$, where $a(t) \neq 0$ for a.e. $t$. Here $N$ is a non-negative integer, and denote those intervals by $\alpha_{i}, i=1,2, \ldots, N$. Similarly, let $\beta_{j}, j=1,2, \ldots, M$, denote the disjoint subintervals of $[0, T]$ such that $a(t)=0$ for a.e. $t \in \beta_{i}$, where $M$ is some non-negative integer. Also let

$$
[0, T]=[a, b] \bigcup\left(\bigcup_{i=1}^{N} \alpha_{i}\right) \bigcup\left(\bigcup_{j=1}^{M} \beta_{j}\right) .
$$

The Grammian matrix can now be written as:

$$
G_{T}=\int_{a}^{b} \Phi(t) B B^{\prime} \Phi^{\prime}(t) d t+\sum_{i=1}^{N} \int_{\alpha_{i}} \Phi(t) B B^{\prime} \Phi^{\prime}(t) d t+\sum_{j=1}^{M} \int_{\beta_{j}} B B^{\prime} d t
$$

If (13) is not satisfied, then neither is $\operatorname{rank} B=n$ satisfied. Thus, according to part (a) of the proof, none of the integrals in (16) is positive definite, i.e. $G_{T}$ is not positive definite ${ }^{1}$ and therefore the system is not completely controllable.

\footnotetext{
1 In this case there exists an orthogonal matrix $V$ such that $V^{\prime} G_{T} V=\left[\begin{array}{cc}G_{1} & 0 \\ 0 & 0\end{array}\right]$, which is clearly not positive definite; the existence of $V$ can easily be derived from the results in [9].
} 
This makes the condition (13) necessary. On the other hand, if (13) does hold, then according to part (a) of the proof, at least the first integral in (16) is positive definite (of course, if $N>0$, then so are all integrals in the intervals $\alpha_{i}$ ). Which means that system (1) is completely controllable. This completes the proof.

\section{Canonical structure}

Kalman canonical decomposition theorem was introduced in [8], [9] (see also [6]), and is a fundamental result in control system theory. When the system is time-varying, the best results so far are those of [24], [25], [26]. Under the assumption of continuous system coefficients, they show that there exists a time-varying transformation matrix that decomposes the system for all time $t$. No systematic procedure for constructing such a transformation matrix is given. The next result shows that a Kalman canonical decomposition of system (1) is possible for all $t$, without continuity assumption on $a(t)$. Moreover, the transformation matrix that achieves this decomposition is time-invariant and can be constructed using the well-established methods (see, e. g. [9], [28]). This is obviously a very rare example of a time-varying system with these features, and should be the first one reported in literature.

For notational simplicity, we consider the decomposition of the state equation only, and the decomposition of the whole system is done similarly.

Theorem 3 Let there exists a number $0<\rho \in \mathbb{R}$ such that $a(t) \neq 0$, a.e. $t \in[0, \rho]$. Then there exists a time invariant transformation matrix $U \in \mathbb{R}^{n \times n}$ that decomposes the system into the completely controllable and uncontrollable parts for all $t>0$.

Proof According to Theorem 2, a necessary condition for not complete controllability is rank $\mathcal{C}=n_{1}<n$. Then, according to [9], there exists a constant matrix $U$ such that

$$
\begin{aligned}
& U^{-1} A U=\left[\begin{array}{cc}
A_{1} & A_{2} \\
0 & A_{3}
\end{array}\right], \quad U^{-1} B=\left[\begin{array}{c}
\bar{B} \\
0
\end{array}\right], \\
& \text { where } A_{1} \in \mathbb{R}^{n_{1} \times n_{1}}, \quad A_{2} \in \mathbb{R}^{n_{1} \times\left(n-n_{1}\right)}, \quad A_{3} \in \mathbb{R}^{\left(n-n_{1}\right) \times\left(n-n_{1}\right)} .
\end{aligned}
$$

Here the pair $\left[A_{1}, \bar{B}\right]$ is completely controllable, whereas the nature of $A_{2}, A_{3}$, is not relevant for our current purposes. By introducing the state transformation $x(t)=U z(t)$, the state equation of (1) is transformed into

$$
\left\{\begin{array}{l}
\dot{z}_{1}(t)=a(t) A_{1} z_{1}(t)+a(t) A_{2} z_{2}(t)+\bar{B} u(t), \\
\dot{z}_{2}(t)=a(t) A_{3} z_{2}(t)
\end{array}\right.
$$

where $z(t)=\left[z_{1}^{\prime}(t), z_{2}^{\prime}(t)\right]^{\prime}$. The state $z_{1}(t)$ is completely controllable for all $t$ due to the fact that $\left[A_{1}, \bar{B}\right]$ is a completely controllable pair, and the fact that if the pair $\left[a(t) A_{1}, \bar{B}\right]$ is completely controllable for some time $T$, it is so for all time $t>0$ (see Theorem 2 ). The state $z_{2}(t)$ is clearly uncontrollable. 


\section{Controller and observer design}

Considerable attention has been given to the problem of stabilisation and state observation of linear time-varying control systems [2], [4], [7], [10], [12], [13], [14], [15], [16], [17], [18], [22], [23]. Common assumptions of these methods are that the system is controllable/observable in a certain sense, and/or derivatives of the coefficients exist up to a certain order. Here we make no such assumptions. Consequently, the existing methods for stabilisation and observation of linear time-varying systems are not applicable to our situation in general. Fortunately, however, it turn out that by a proper modification, methods of linear time-invariant systems can be used. We begin by considering the problem of stabilisation of (1) by means of linear state-feedback controller, and then use such a result to design the state observer. The following is a standing assumption of this section.

Assumption (A1). There exists a number $0<\rho \in \mathbb{R}$ such that $a(t) \neq 0$, a.e. $t \in[0, \rho]$, and

$$
\lim _{t \rightarrow \infty} \int_{0}^{t} a(\tau) d \tau= \pm \infty
$$

Recall that the pair of matrices $[a(t) A, B]$ is stabilisable if there exist a matrix $K(t)$ such that the system $\dot{x}(t)=[a(t) A+B K(t)] x(t)$ is asymptotically stable. Similarly, the pair $[a(t) A, C]$ is detectable if the pair $\left[a(t) A^{\prime}, C^{\prime}\right]$ is stabilisable.

Theorem 4 (a) If $\lim _{t \rightarrow \infty} \int_{0}^{t} a(\tau) d \tau=\infty$, then the pair $[a(t) A, B]$ is stabilisable if and only if the eigenvalues of the matrix $A_{3}$ in the Kalman decomposition (17) have negative real parts.

(b) If $\lim _{t \rightarrow \infty} \int_{0}^{t} a(\tau) d \tau=-\infty$, then the pair $[a(t) A, B]$ is stabilisable if and only if the eigenvalues of the matrix $A_{3}$ in the Kalman decomposition (17) have positive real parts.

Proof We prove part (b) only, since the proof of part (a) is done similarly. Due to assumption $A 1$ and Theorem 3, there exists a constant matrix $U$ through which the state equation of (1) can be decomposed into (18) using the transformation $x(t)=U z(t)$. Since $U$ is a constant matrix, the stability of $x(t)$ is equivalent with the stability of $z(t)$. Hence we focus on the stabilisability of (18). The solution of the uncontrollable system $\dot{z}_{2}(t)=a(t) A_{3} z_{2}(t)$ is

$$
z_{2}(t)=\exp \left[A_{3} \int_{0}^{t} a(\tau) d \tau\right] z_{2}(0)
$$

Clearly, $\lim _{t \rightarrow \infty} z_{2}(t)=0, \forall z_{2}(0) \in \mathbb{R}^{n_{1} \times n_{1}}$, if and only if all eigenvalues of $A_{3}$ have positive real parts. On the other hand, since the pair $\left[A_{1}, \bar{B}\right]$ is completely controllable, by Wonham's theorem [27], there exists $K_{1} \in \mathbb{R}^{m \times\left(n-n_{1}\right)}$ such that the matrix $A_{1}+\bar{B} K_{1}$ has any desired set of eigenvalues. We choose the control $u(t)=a(t) K_{1} z_{1}(t)$, where $K_{1}$ is such that all the eigenvalues of 
$A_{1}+\bar{B} K_{1}$ have positive real parts. The system (18) under this control becomes $\dot{z}(t)=a(t) \bar{A} z(t)$ where

$$
\bar{A}=\left[\begin{array}{cc}
A_{1}+\bar{B} K_{1} & A_{2} \\
0 & A_{3}
\end{array}\right] .
$$

Since all eigenvalues of $\bar{A}$ have positive real parts, the system (18) is asymptotically stable.

It is interesting to note that the stabilisability of (1) does not depend on the particular form of $a(t)$ but only on the behavior of its integral at infinity. In part (a) of the above theorem, the conditions for stabilisability are the same as for the time-invariant system. However, these conditions in part (b) are very different from the corresponding ones of time-invariant system as it requires the eigenvalues of $A$ to have positive real parts. For the rest of the section we make the following minimum assumption on the system.

Assumption (A2). The pair $[a(t) A, B]$ is stabilisable, and the pair $[a(t) A, C]$ is detectable.

While Theorem 4 gives necessary and sufficient conditions for stabilisability, its proof gives the main idea for constructing a linear state-feedback stabilising controller of the form $u(t)=K(t) x(t)$. We first need to design the matrix $K_{1}$ such that $A_{1}+\bar{B} K_{1}$ has the desired eigenvalues. This can be done by any of the well-known methods of linear time-invariant systems (see, e. g. [5]). Decomposing the matrix $U^{-1}$ in block form as:

$$
U^{-1}=\left[\begin{array}{ll}
U_{1} & U_{2} \\
U_{3} & U_{4}
\end{array}\right],
$$

with $U_{1}$ being of dimension $\left(n-n_{1}\right) \times\left(n-n_{1}\right)$, we can write the stabilising feedback gain as

$$
K(t)=a(t) K_{1}\left[\begin{array}{ll}
U_{1} & U_{2}
\end{array}\right] .
$$

We now focus on the design of a full order Luenberger observer, and one can easily adopt this approach to design the reduced order observer. Consider the following linear time-varying observer:

$$
\left\{\begin{array}{l}
\dot{\hat{x}}(t)=a(t) A \widehat{x}(t)+B u(t)+L(t)[y(t)-\widehat{y}(t)], \\
\widehat{y}(t)=C \widehat{x}(t)
\end{array}\right.
$$

The matrix $L(t)$ of order $n \times p$ needs to be such that $\lim _{t \rightarrow \infty} e(t)=0$, where $e(t) \equiv x(t)-\widehat{x}(t)$ is the state estimation error. The equation of this error is:

$$
\dot{e}(t)=[a(t) A-L(t) C] e(t) .
$$

Since the pair $[a(t) A, C]$ is detectable, there exists a matrix $L(t)$ such that (23) is asymptotically stable. Of course, $L(t)$ can be determined using the previous 
procedure of designing $K(t)$. Next we show that the system consisting of the controller that feeds back the state of the observer, is asymptotically stable. Let the controller be chosen as a linear feedback from the estimated state as:

$$
u(t)=K(t) \widehat{x}(t)=K(t)[x(t)-e(t)] .
$$

where $K(t)$ is given in (21). The state equation now becomes:

$$
\dot{x}(t)=[a(t) A+B K(t)] x(t)-a(t) B K(t) e(t) .
$$

Denoting by $s(t) \equiv\left[x^{\prime}(t), e^{\prime}(t)\right]^{\prime}$, we have that $\dot{s}(t)=H(t) s(t)$, where

$$
H(t)=\left[\begin{array}{cc}
a(t) A+B K(t) & -B K(t) \\
0 & a(t) A-L(t) C
\end{array}\right],
$$

which is clearly an asymptotically stable system.

\section{Conclusions}

We have derived several basic results for a class of linear time-varying systems. These are: an algebraic criterion for complete controllability, canonical decomposition of the system for all time $t$, and a design approach to stabilising controllers and observers. Despite the weak assumptions on the time-varying coefficient, the results are very explicit and have a strong link with the rich theory of linear time-invariant systems. It would be interesting to investigate if more general linear time-varying systems can be approximated by or transformed into this class of systems.

\section{Acknowledgement}

The authors are grateful to the Reviewer for a careful reading of their article, and for several very useful remarks which have lead to a significantly improved version of the paper.

\section{References}

1. Athreya, K. B., Lahiri, S. N., Measure theory and probability theory, Springer, 2006

2. Chai, W., and Loh, N. K., Design of minimal-order state observers for time-varying multivariable systems, I. J. of Systems Science, 23, 4, pp. 581-592, 1992.

3. Chang, A., An algebraic characterization of controllability, IEEE Trans. Automatic Control, AC-10, pp. 112-113, 1965.

4. Chen, M.-S., and Yen, J.-Y., Application of the least squares algorithm to the observer design for linear time-varying systems, IEEE Trans. Automatic Control, 44. 9., pp. $1742-$ $1745,1999$.

5. D'Azzo, J. J., and Houpis, C. H., Linear control system analysis and design: conventional and modern, Third Edition, McGraw-Hill book Company, 1988.

6. Gilbert, E. G., Controllablity and observability in multivariable control systems, SIAM J. Control, Ser A, Vol. 1, No. 2, pp. 128-151, 1963. 
7. Kalman, R. E., Contributions to the theory of optimal control, Boletin-Sociedad Matematica Mexicana, pp. 102-119, 1960.

8. Kalman, R. E., Canonical structure of linear dynamical systems, Proc. Nat. Acad. Sci. USA, 48, pp. 596-600.

9. Kalman, R. E., Mathmatical description of linear dynamical systems, SIAM J. Control, Ser A, Vol. 1, No. 2, pp. 152-192, 1963.

10. Lee, H. C., and Choi, J. W., Ackermann-like eigenvalue assignment formulae for linear time-varying systems, IEE Proc.-Control Theory Appl., 152, 4, pp. 427-434, 2005.

11. Leiva, H., and Zambrano, H., Rank condition for the controllablity of linear time-varying system, Int. J. Control, 72, 10, pp. 929-931, 1999.

12. Lovass-Nagy, V., Miller, R. J., and Mukundan, R., On the application of matrix generalized inverses to the design of observer for time-varying and time-invariant linear systems, IEEE Trans. Automatic Control, AC-25, 6, pp. 1213-1218, 1980

13. Malek-Zavarei, M., The stability of linear time-varying systems, I. J. Control, 27, 5, pp. 809-815, 1978.

14. Nguyen, C., and Lee, T. N., Design of a state estimator for a class of time-varying mutivariable systems, IEEE Trans. Automatic Control, AC-30, 2, pp. 179-182, 1985.

15. Nguyen, C. C., Canonical transformation for a class of time-varying multivariable systems, I. J. Control, 43, 4, pp. 1061-1074, 1986.

16. Nguyen, C. C., Arbitrary eigenvalue assignments for linear time-varying multivariable control systems, I. J. Control, 45, 3, pp. 1051-1057, 1987.

17. Nguyen, C. C., Design of reduced-order state estimators for linear time-varying multivariable systems, I. J. Control, 46, 6, pp. 2113-2126, 1987.

18. Phat, V. N., Global stabilization for linear continuous time-varying systems, Applied mathematics and computation, 175, pp. 1730-1743, 2006.

19. Silverman, L. M. and Meadows, H. E., Controllability and observability in time-variable linear systems, SIAM J. Control, 5, pp. 64-73, 1967.

20. Sontag, E. D., Mathematical control theory: deterministic finite dimensional systems, Second Edition, Springer, 1998.

21. Stubberud, A. R., A controllablity criterion for a class of linear systems, IEEE Trans. Applications and Industry, 68, pp. 411-413, 1964.

22. Tsui, C. C., Function-observer design for a class of linear time-varying system, I. $J$. Control, 44, 1, pp. 277-282, 1986.

23. Valášek, and Olgac, N., Efficient eigenvalue assignment for general linear MIMO systems, Automatica, 31, 11, pp. 1605-1617, 1995.

24. Weiss, L., and Kalman, R. E., Contributions to linear system theory, Int. J. of Eng. Sci., 3, pp. 141-171, 1965.

25. Weiss, L., On the sturucture theory of linear differential systems, SIAM J. Control, Vol. 6, No. 4, pp. 659-680, 1968.

26. Weiss, L., and Falb, P. L., Doležal's theorem, linear algebra with continuously parametrized elemnets, and time-varying systems, Math. System Theory, 3, pp. 67-75, 1969.

27. Wonham, W. M., On pole assignment in multi-input controllable linear systems, IEEE Trans. of Automatic Control, AC-12, 6, pp. 660-665, 1967.

28. Zhou, K., Doyle, J. C., and Glover, K., Robust and optimal control, Prentice Hall, 1996. 